Supporting Information for

\title{
Half-time heat map reveals ultrasonic effects on morphology and kinetics of amyloidogenic aggregation reaction
}

Kichitaro Nakajima†, Hajime Todađ, Keiichi Yamaguchi†, Masatomo So ${ }^{\ddagger}$, Kensuke Ikenaka§, Hideki Mochizuki ${ }^{\S}$, Yuji Goto ${ }^{\dagger}$, and Hirotsugu Ogi*, ${ }^{\star}$

$\uparrow$ Global Center for Medical Engineering and Informatics, Osaka University, Suita, Osaka 565-0871, Japan

- Graduate School of Engineering, Osaka University, Suita, Osaka 565-0871, Japan

$\$$ Astbury Centre for Structural Molecular Biology, University of Leeds, Leeds LS2 9JT, UK

$\S$ Department of Neurology, Graduate School of Medicine, Osaka University, Suita, Osaka 565-0871, Japan

*E-mail: ogi@prec.eng.osaka-u.ac.jp

Table of Content

SI Appendix 1: Page S2

Fig. S1: Page S3

SI Appendix 2: Fig. S2: Page S4

Fig. S3: Page S5

SI References: Page S6 


\section{SI Appendix 1. Reproducible amyloid-fibril assay using laboratory-built ultrasonic instrument}

In the amyloid-fibril assays, the reaction kinetics is often discussed using the half time (i.e., $t_{\text {half }}{ }^{[1,2]}$, which is the time when the ThT fluorescence intensity becomes the half its maximum. Because the $t_{\text {half }}$ value varies widely even among the identical assays due to the high energy barrier for the nucleation ${ }^{[3]}$, achieving a high reproducibility is challenging in an amyloid-fibril assay. Especially for ultrasonication, precise control of the fibril-formation reaction is very difficult because the reaction is highly sensitive to the acoustic field ${ }^{[3,4]}$. We here improved the ultrasonic instrument developed previously ${ }^{[2]}$ to overcome the difficulty in the reproducibility of the assay as demonstrated below.

The fibril-formation reaction of the $\beta 2 \mathrm{~m}$ monomer solution is monitored with a timecourse of the ThT fluorescence intensity as shown in Figure S1. Under quiescence, the ThT intensity starts increasing after a 20 -h lag time and reaches a plateau at $40 \mathrm{~h}$. The AFM image of a sample incubated for $50 \mathrm{~h}$ under quiescence shows the typical fibril morphology (Figure $2 \mathrm{e}$, Main text). By shaking, the lag time is shortened to $\sim 10 \mathrm{~h}$. In addition, the ThT intensity precipitously increases after the lag time compared to quiescence. The resultant fibrils are shown in Figure $2 f$ (Main text). The lag phase and following evolution phase correspond to the nucleation and fibril elongation phases, respectively ${ }^{[5]}$. Thus, shaking accelerates both the nucleation and elongation reactions of the fibril formation. By ultrasonication, the fibril formation is further accelerated. The resultant fibrils are shorter than those formed under quiescence and shaking (Figure 2g, Main text). The difference in the morphology of the formed aggregates is discussed in detail in the following section.

The $t_{\text {half }}$ value of the quiescent assay is $31.5 \pm 0.2 \mathrm{~h}$, which varies with the coefficient of variation $(\mathrm{CV})$ value of $0.6 \%$. The $\mathrm{CV}$ value is significantly smaller than those with agitations: The $\mathrm{CV}$ value of the shaking assay is $10 \%$ ( $\left.t_{\text {half }}=11.1 \pm 1.1 \mathrm{~h}\right)$. For ultrasonication, our previous value was $22 \%{ }^{[2]}$, being less reproducible than shaking. In this study, we adopted the acoustic couplant to improve the stability of the contact between the PZT transducer and plastic film on the sample solutions. Also, we removed external forces applied to each transducer for the acoustical contact except for its own weight. Consequently, the ultrasonication assay shows the $t_{\text {half }}$ value of $6.8 \pm 0.8 \mathrm{~h}(\mathrm{CV}=12 \%)$ in this study. This improved $\mathrm{CV}$ value of the ultrasonication assay is comparable to that in the shaking assay, contributing to the following systematic investigation of the aggregation reactions. 


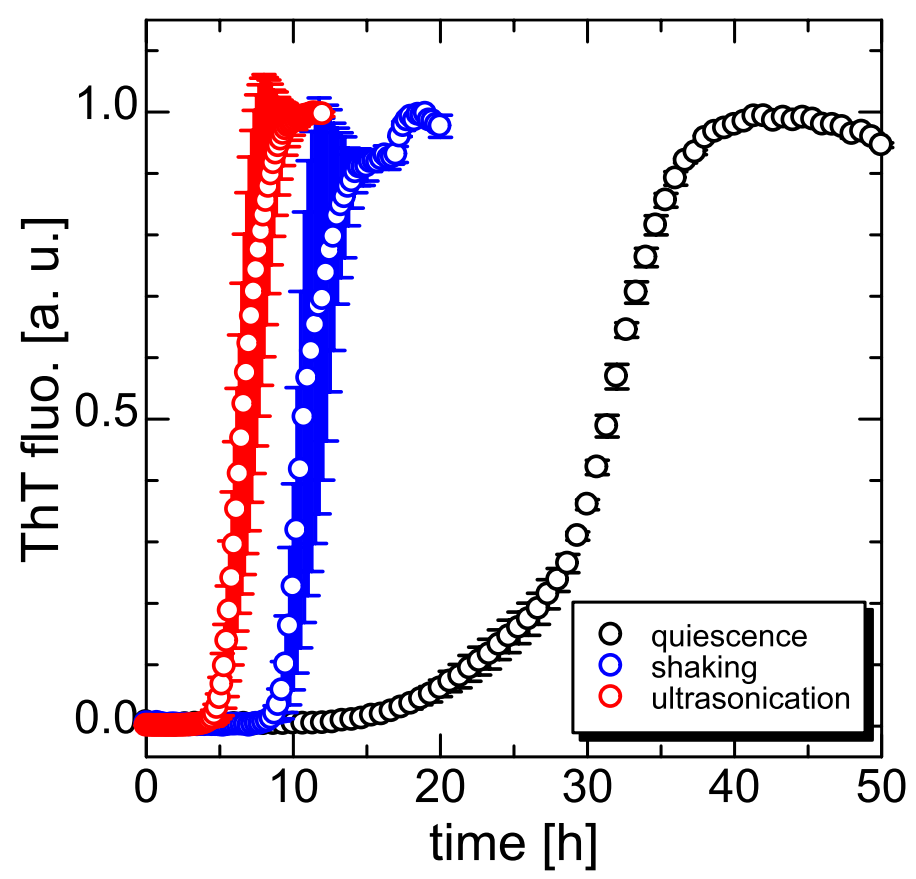

Figure S1. Time-course curves of the ThT fluorescence intensity of the $0.3-\mathrm{mg} / \mathrm{mL} \beta 2 \mathrm{~m}$ monomer solutions with $150-\mathrm{mM} \mathrm{NaCl}$ with quiescence (black circles), shaking (blue circles), and ultrasonication (red circles). The ThT fluorescence measurement was performed for the multiple solutions $(n>3)$. The error bars denote a standard deviation of the ThT intensity at each time among multiple solutions $(n>3)$. 


\section{SI Appendix 2. Detailed analysis of the AFM images}

\section{(a) $\mathrm{Q} / 80 \mathrm{mM}$}
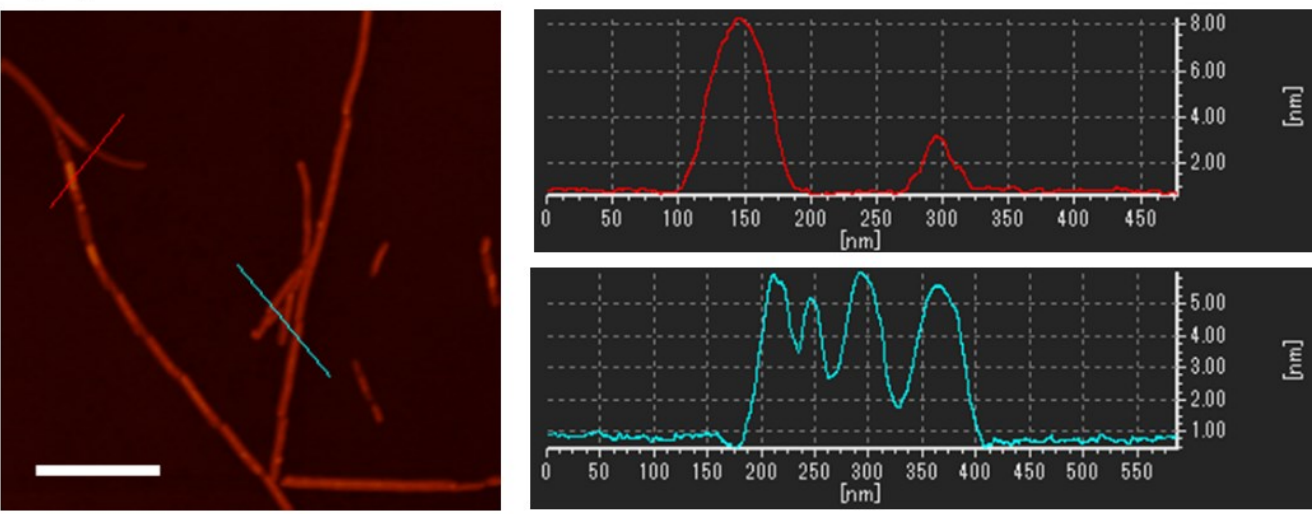

\section{(b) $\mathrm{S} / 80 \mathrm{mM}$}
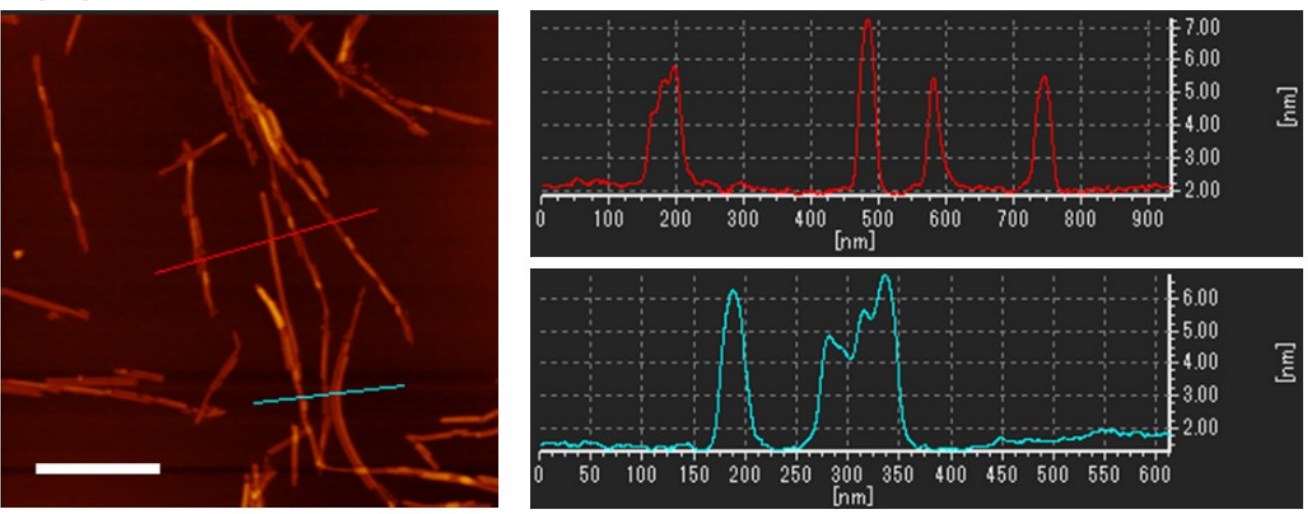

\section{(c) US / $80 \mathrm{mM}$}
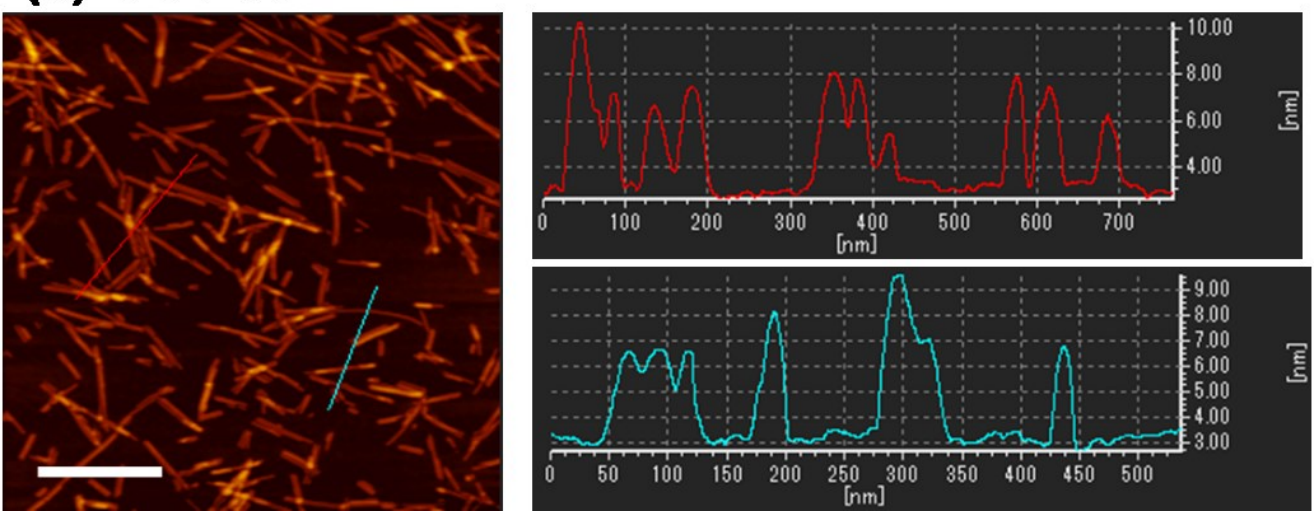

Figure S2. AFM images of the fibrils formed in the solution with the salt concentration of 80 mM under (a) quiescence, (b) shaking, and (c) ultrasonication, respectively. For each sample, the left panel shows the AFM image with a 500-nm scale bar. The red and cyan lines indicate the lines analyzed their cross-sectional profile as shown in the right panels. Under all agitations, the fibrils with the height between 5 and $10 \mathrm{~nm}$ formed. 


\section{(a) S / $240 \mathrm{mM}$}
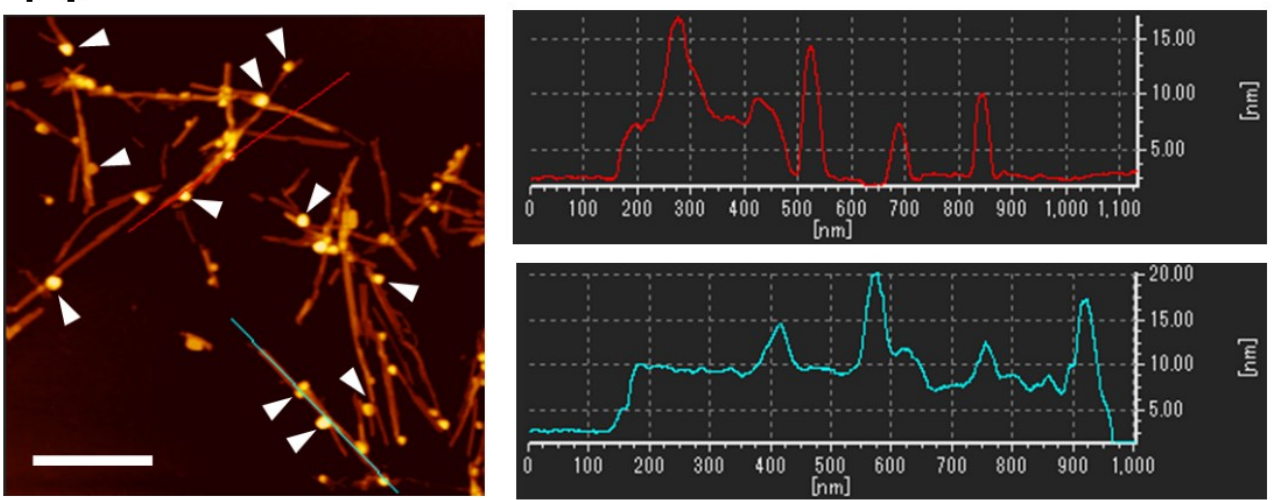

\section{(b) $\mathrm{S} / 480 \mathrm{mM}$}
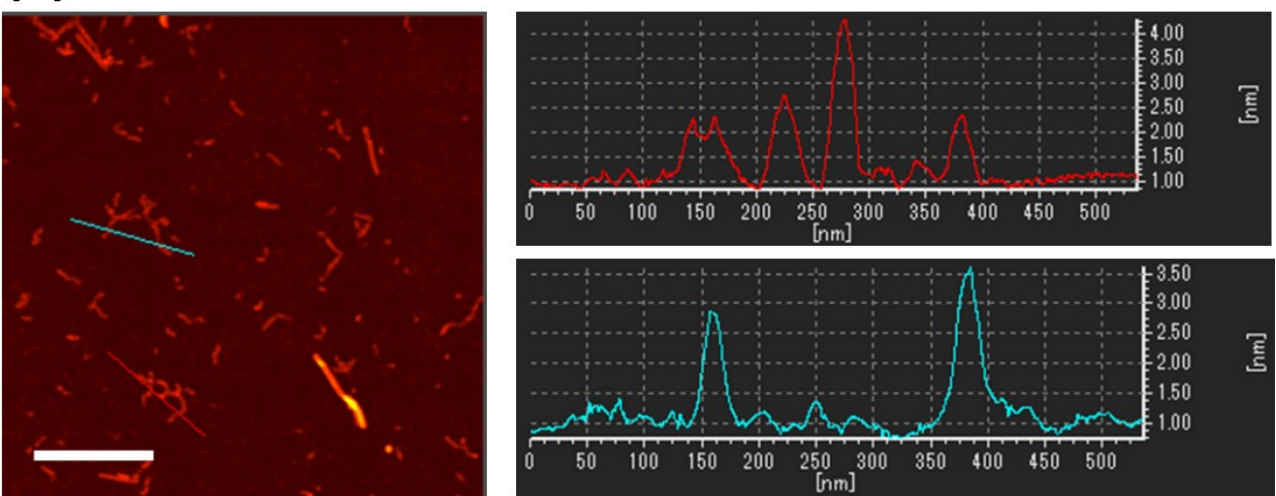

\section{(c) $\mathrm{Q} / 480 \mathrm{mM}$}
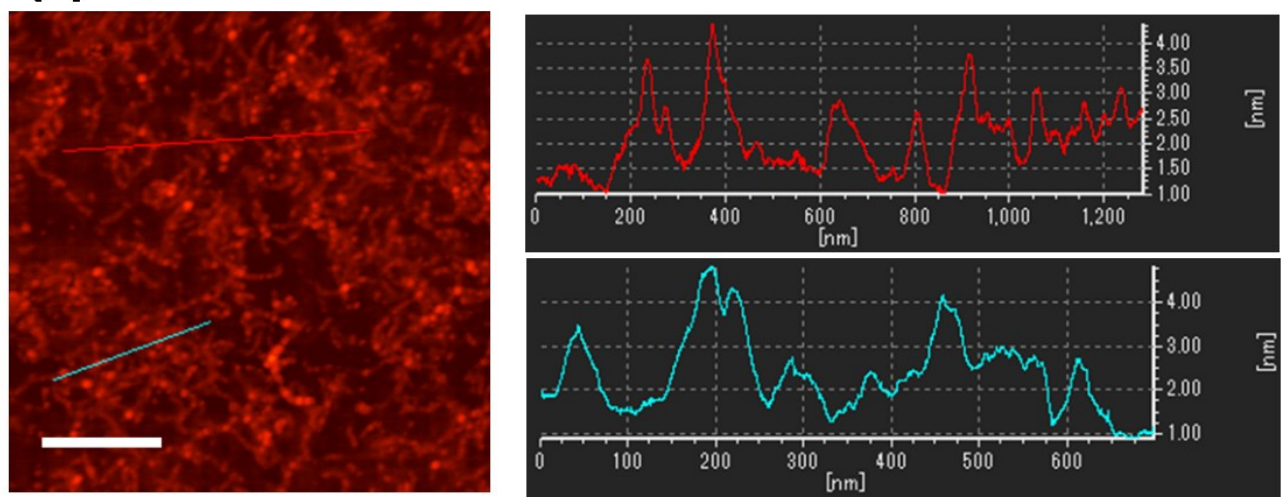

Figure S3. (a) The AFM image of the aggregates formed in the solution with the salt concentration of $240 \mathrm{mM}$ under shaking. The white arrows in the left panel indicate the globular aggregates attached on the fibril surface. As shown in the right panels, the globular aggregates with the height of $\sim 10 \mathrm{~nm}$ are attached on the fibrils with the height $\sim 8 \mathrm{~nm}$. (b), (c) The AFM images of the amorphous aggregates (curvilinear fibrils) formed in the solution with the salt concentration of $480 \mathrm{mM}$ under (b) shaking and (c) quiescence. The height of the aggregates is less than $4 \mathrm{~nm}$, being different from the fibrils shown in Figure S2. 


\section{SI References}

[1] Shahnawaz, M.; Tokuda, T.; Waragai, M.; Mendez, N.; Ishii, R.; Trenkwalder, C.; Mollenhauer, B.; Soto, C. Development of a Biochemical Diagnosis of Parkinson Disease by Detection of a-Synuclein Misfolded Aggregates in Cerebrospinal Fluid. JAMA Neurol. 2017, 74, 163-172.

[2] Nakajima, K.; Noi, K.; Yamaguchi, K.; So, M.; Ikenaka, K.; Mochizuki, H.; Ogi, H.; Goto, Y. Optimized Sonoreactor for Accelerative Amyloid-Fibril Assays through Enhancement of Primary Nucleation and Fragmentation. Ultrason. Sonochem. 2021, 73, 105508.

[3] Umemoto, A.; Yagi, H.; So, M.; Goto, Y. High-throughput Analysis of UltrasonicationForces Amyloid Fibrillation Reveals the Mechanism Underlying the Large Fluctuation in the Lag Time. J. Biol. Chem. 2014, 289, 27290-27299.

[4] Nakajima, K.; Ogi, H.; Adachi, K.; Noi, K.; Hirao, M.; Yagi, H.; Goto, Y. Nucleus Factory on Cavitation Bubble for Amyloid $\beta$ Fibril. Sci. Rep. 2016, 6, 22015.

[5] Morris, A. M.; Watzky, M. A.; Agar, J. N.; Finke, R. G. Fitting Neurological Protein Aggregation Kinetic Data via a 2-step, Minimal/"Ockham's Razor" Model: the Finke-Watzky Mechanism of Nucleation followed by Autocatalytic Surface Growth. Biochemistry 2008, 47, 2413-2427. 